\title{
Sistema de previsão da safra de soja para o Brasil
}

\author{
Eduardo Delgado Assad(1), Fábio Ricardo Marin ${ }^{(1)}$, Silvio Roberto Evangelista(1), Felipe Gustavo Pilau(1), \\ José Renato Bouças Farias( ${ }^{(2)}$, Hilton Silveira Pinto ${ }^{(3)}$ e Jurandir Zullo Júnior ${ }^{(3)}$
}

\begin{abstract}
(1)Embrapa Informática Agropecuária, Av. Dr. André Tosello, 209, Cidade Universitária, Caixa Postal 6041, CEP 13083-886 Campinas, SP. E-mail: assad@cnptia.embrapa.br, marin@cnptia.embrapa.br, silvio@cnptia.embrapa.br, felipe@cnptia.embrapa.br (2)Embrapa Soja, Rod. Carlos João Strass, Distrito de Warta, Caixa Postal 231, CEP 86001-970 Londrina, PR. E-mail: jrenato@cnpso.embrapa.br (3)Centro de Ensino e Pesquisa em Agricultura, Av. Dr. André Tosello, 209, Cidade Universitária, CEP 13083-970 Campinas, SP. E-mail: hilton@cpa.embrapa.br, jurandir@cpa.embrapa.br
\end{abstract}

Resumo - Este trabalho teve por objetivo avaliar um sistema de previsão de safra de soja para o Brasil, baseado em modelos empíricos regionalizados para estimativa da produtividade, a partir de um banco de dados de área cultivada em escala municipal, e de um sistema de monitoramento agrometeorológico de abrangência nacional. Os modelos incorporam a base conceitual proposta por Doorenbos \& Kassam, com ajustes empíricos para cada região do Brasil, considerando-se as diferenças quanto ao potencial produtivo das principais variedades e as peculiaridades dos sistemas de produção utilizados nas diferentes regiões. A base de informações de área cultivada de cada Estado foi constituída por dados provenientes do IBGE e da Conab. A produção de soja, entre as safras 2000/2001 e 2005/2006, foi estimada e comparada com os levantamentos da Conab. A análise estatística pelo teste $t$ indica não haver diferença entre as estimativas e os dados oficiais. Bons ajustes $\left(R^{2} \geq 0,87\right)$ foram obtidos para as produções regionalizadas, com desvios mais expressivos nos estados do Rio Grande do Sul, Santa Catarina, Mato Grosso do Sul, Maranhão, Piauí e Bahia. Para os dados consolidados em escala nacional, o maior desvio observado foi de $5,81 \%$, na safra 2000/2001, e o menor de $0,62 \%$ na safra 2005/2006.

Termos para indexação: produtividade, modelagem, tecnologia da informação, agrometeorologia.

\section{Forecast system of soybean crop yield for Brazil}

\begin{abstract}
The aim of this work was to evaluate a system to forecast soybean crop yield for Brazil, based on regional empirical models to assess crop yield, with data from a national database of soybean cropped area in municipal scale, and from an agrometeorological monitoring system covering all Brazilian States. The models incorporated the conceptual bases proposed by Doorenbos \& Kassam, with empirical adjustments for each region of Brazil, considering the diverse potential yield of most used varieties and the regional differences in the soybean production systems. Cultivated area database from each State was structured with data from IBGE and Conab. Soybean yield was estimated for harvests from 2000/2001 to 2005/2006 and compared to Conab surveys. Statistical analysis using Test $t$ point out that there is no significant difference between estimates and official data. Good fittings were gotten for region grouped yield data $\left(R^{2} \geq 0.87\right)$, with higher deviations for harvest assessment of Rio Grande do Sul, Santa Catarina, Mato Grosso do Sul, Maranhão, Piauí and Bahia. In national scale, the highest observed deviation was $5.81 \%$ for the $2000 / 2001$ harvest, and the smaller one was $0.62 \%$ for the 2005/2006 yield.
\end{abstract}

Index terms: crop yield, modeling, information technology, agrometeorology.

\section{Introdução}

A estimativa da safra agrícola de um país e o conhecimento da sua distribuição no espaço geográfico são de extrema importância para o planejamento estratégico do Estado, no que concerne à formulação de políticas públicas, à logística e à segurança alimentar, além de atuar como elemento importante na formação de preços nos mercados interno e externo (Figueiredo, 2005).
Para a estimativa da produção, é necessário conhecer a área cultivada com a espécie de interesse e a sua produtividade. Uma das formas para o levantamento da área cultivada é a aplicação de técnicas de geoprocessamento e sensoriamento remoto às imagens de satélites (Antunes, 2005; Rizzi \& Rudorff, 2005; Rudorff et al., 2005) e, também, pelo uso da amostragem estratificada em associação com dados orbitais (Epiphanio et al., 2002; Luiz et al., 2002). Em escala 
operacional, contudo, o levantamento da área cultivada ainda se baseia em métodos subjetivos, utilizados pela Companhia Nacional de Abastecimento (Conab) e pelo Instituto Brasileiro de Geografia e Estatística (IBGE), órgãos envolvidos na produção e divulgação dos dados oficiais da área e produção agrícola no território nacional.

A estimativa de produtividade com base em modelos agrometeorológicos é complexa, especialmente quando se pretende realizar estimativas em macroescala e consolidar seus resultados, para a obtenção de números que representem a realidade do país. Modelos de simulação agrícola podem ser entendidos como equações matemáticas, empíricas ou mecanísticas, que visam simplificar a realidade e representar o acúmulo de biomassa e o desenvolvimento das plantas, para antever sua produtividade em função dos fatores influentes (Acock \& Acock, 1991; Passioura, 1996; CheerooNayamuth, 1999; Pedersen \& Lauer, 2002). Entre as culturas agrícolas de destaque mundial, a soja (Glycine $\max ($ L.) Merr.) tem sido objeto de um grande número de estudos, que buscam compreender e quantificar as relações ecofisiológicas que interferem na formação da produção da cultura (Sinclair et al., 1986; Acock \& Trent, 1991; Hoogenboom et al., 1994; Reddy et al., 1995; Board et al., 1996; Sau et al., 1999; Alagarswamy et al., 2000; Garcia-Paredes et al., 2000; Ruiz-Nogueira et al., 2001; Board, 2002; Popp et al., 2003).

Os modelos propostos por Jensen (1968) e Doorenbos \& Kassam (1979) relacionam a depleção da produtividade à sensibilidade das culturas ao estresse hídrico, nas várias fases de desenvolvimento das plantas. Ambos são compostos por dois módulos para estimativa da produtividade - um que trata do potencial produtivo regional e outro que estima a produtividade real - e são mais indicados para aplicações em escala macroclimática (Ferraudo et al., 1995; Moraes et al., 1998; Fontana et al., 2001; Berka et al., 2003).

Outra dificuldade associada à estimativa da produtividade é a sua adequada representação espacial. Os modelos agrometeorológicos integram o acúmulo (ou a perda) de biomassa das culturas ao longo do tempo, utilizando dados das estações meteorológicas de superfície. Tem-se, portanto, resultados pontuais, que precisam ser interpolados para a obtenção das estimativas em escala estadual e nacional. A geração de mapas é facilitada, quando se dispõem de modernos recursos da tecnologia da informação, que permitem o tratamento e a espacialização de dados pontuais para a composição de mapas (Romani et al., 2003).
Considerando-se a importância da cultura da soja ao agronegócio brasileiro, e a necessidade de se dispor de um sistema que permitisse estimar com certa antecedência a produção nacional, teve-se por objetivo, neste trabalho, avaliar um sistema de previsão de safra de soja para o Brasil, baseado em modelos empíricos regionalizados para estimativa da produtividade, a partir de um banco de dados de área cultivada em escala municipal e de um sistema de monitoramento agrometeorológico de abrangência nacional.

\section{Material e Métodos}

O sistema de previsão de produtividade de soja para o Brasil (SPSoja-Agritempo) incorpora a estimativa da produtividade potencial (Pp), baseada no método das zonas agroecológicas (MZA) (Doorenbos \& Kassam, 1979), que pressupõe que as exigências hídricas, nutricionais e fitossanitárias da cultura sejam atendidas, e que a produtividade seja condicionada apenas pelas características da cultura e pelas condições ambientais, representadas pela radiação solar, fotoperíodo e temperatura do ar. Ajustes empíricos podem ser feitos tanto ao módulo de estimativa da produtividade potencial, como ao módulo de produtividade real, para adaptação dos valores à realidade de cada região. A Equação (1) descreve conceitualmente o MZA:

$\mathrm{Pp}=\mathrm{Fb}\left[\mathrm{C}_{\mathrm{IAF}} \cdot \mathrm{C}_{\mathrm{RES}} \cdot \mathrm{C}_{\mathrm{COL}} \cdot \mathrm{NDC} /(1+\mathrm{U})\right]$

em que $\mathrm{Fb}$ é a fotossíntese bruta de uma cultura hipotética padrão $\left(\mathrm{t} \mathrm{ha}^{-1} \mathrm{dia}^{-1}\right)$, calculada em função da disponibilidade de radiação solar e da temperatura do ar; $\mathrm{C}_{\mathrm{IAF}}$ é a correção da fotossíntese, em função da variação da área foliar da cultura, calculada por uma função de segundo grau, que tem o IAF como variável independente; $\mathrm{C}_{\mathrm{RES}}$ é a correção da taxa de manutenção da cultura (respiração), em função da temperatura do ar entre $0,5\left(\mathrm{Temp}<20^{\circ} \mathrm{C}\right)$ e $0,6\left(\mathrm{Temp} \geq 20^{\circ} \mathrm{C}\right) ; \mathrm{C}_{\mathrm{COL}}$ é a correção para o índice de colheita; U é a correção para a umidade da parte colhida, para conversão do peso seco em peso fresco; e NDC é a duração do período de desenvolvimento da cultura.

A produtividade real (Yp) é estimada a partir da deficiência hídrica relativa [1 - (ER/EP)], ponderada por um fator de sensibilidade da cultura ao deficit hídrico (ky), conforme Doorenbos \& Kassam (1979):

$\mathrm{Yr} / \mathrm{Yp}=1$ - ky[1- (ER/EP)]

em que Yr é a produtividade estimada; Yp é a produtividade potencial; ER é a evapotranspiração real da cultura; EP é a evapotranspiração potencial da cultura, 
estimada pelo método de Thornthwaite (1948), e o coeficiente de cultura $(\mathrm{kc})$ para cada fase fenológica; e ky é o coeficiente de penalização da produtividade por deficit hídrico, variável com a fase fenológica da cultura.

A evapotranspiração real da cultura (ETR) foi determinada pelo balanço hídrico seqüencial, segundo Thornthwaite \& Mather (1955). Os cálculos de balanço hídrico utilizam a base de dados diários de temperatura do ar e chuva, provenientes de 1.053 estações meteorológicas espalhadas por todo território nacional, pertencentes a diversas instituições governamentais ou privadas, em acordo com o Sistema de Monitoramento Agrometeorológico (Agritempo), que disponibiliza os dados meteorológicos diariamente.

Ao modelo de estimativa de produtividade real, proposto por Doorenbos \& Kassam (1979), foram acrescentados coeficientes empíricos de ajuste, específicos para cada região do país, para contemplar as especificidades regionais das variedades e as particularidades dos sistemas de produção empregados. Essa especificação regional empírica do modelo foi feita com os dados de produtividade obtidos de avaliação de cultivares (Reunião..., 2004).

Apesar dos grandes avanços científicos no uso do geoprocessamento de imagens de satélite, em estimativa de área de culturas agrícolas, ainda não se dispõe de um método operacional e de baixo custo, para estimativa nacional da área plantada de soja. Alternativamente, para se estimar a safra nacional de soja, além da estimativa da produtividade o sistema incorpora um módulo de acesso a um banco de dados, baseado nos levantamentos feitos pelo Instituto Brasileiro de Geografia e Estatística (IBGE), em escala municipal. O sistema aponta os municípios produtores de soja e associa a cada um deles sua fração relativa à área total de soja produzida. Na escala estadual, são utilizados os dados de área plantada, provenientes de levantamentos feitos pela Companhia Nacional de Abastecimento (Conab), que são convertidos em áreas municipais, multiplicando-se a fração relativa do município pela área total do Estado.

O SPSoja-Agritempo calcula a produtividade real para cada estação meteorológica de superfície. Os valores de produtividade calculados pontualmente são, então, interpolados e multiplicados pela área cultivada na escala municipal, totalizada posteriormente para o Estado. A produtividade média estadual é obtida pela simples relação entre a produção e a área total do Estado. O Sistema não disponibiliza as produções municipais de soja em função do modelo agrometeorológico adotado e da atual base de dados de área plantada disponível.

O SPSoja-Agritempo utiliza a base computacional do Sistema Agritempo que, por sua vez, foi desenvolvido com arquitetura Web, em plataforma Java, e a tecnologia Java Servlet, com acesso a banco de dados via JDBC. Os servidores utilizados no sistema são Apache HTTP Server (Web Server), Apache Tomcat (Servlet Engine) e Banco de Dados Oracle 9i.

Para a avaliação do sistema de previsão e monitoramento da safra de soja para o Brasil, procedeuse às estimativas das produções de soja para as safras 2000/2001, 2001/2002, 2002/2003, 2003/2004 e 2004/ 2005. Estimativas da produção de soja para o ano agrícola 2005/2006 foram realizadas ao longo da safra, e buscou-se avaliar a capacidade do sistema em estimar a produção com antecedência à colheita, nos meses de janeiro, março e maio - quando a colheita já havia sido praticamente encerrada, o que coincidiu com as divulgações dos 3o, 4o e 6o levantamentos de avaliação da safra 2005/2006 (Conab, 2006b). Para avaliação do desempenho do SPSoja-Agritempo, adotaram-se os valores de produção e produtividade divulgados pelos boletins de levantamento de safra da Conab, pois os dados de área de plantio usados provieram do referido órgão governamental, que são a referência do governo brasileiro (Conab, 2006a). Os valores estimados e oficiais das produções estaduais de soja foram submetidos à analise estatística pelo uso do teste t, a $5 \%$ de probabilidade.

\section{Resultados e Discussão}

As probabilidades $\mathrm{P}(\mathrm{T} \leq \mathrm{t})$ resultantes da análise estatística pelo uso do teste $t$ não indicaram diferenças significativas, quando comparadas as produções estaduais de soja estimadas pelo SPSojaAgritempo aos valores oficiais divulgados pela Conab, para as seis safras avaliadas. Apesar de não apresentarem diferença, nos estados de Mato Grosso do Sul e Tocantins, foram observadas as menores probabilidades no teste $t$, o que indica que os desvios entre a estimativa e a referência foram relativamente maiores que as observadas nos demais Estados. Ainda, pela análise dos valores de r, pode-se observar que as oscilações das produções estaduais de soja, de uma safra agrícola à outra, são muito semelhantes, à exceção dos estados de Santa Catarina e da Bahia (Tabela 1). 
Na Região Sul, que contempla o segundo (Paraná) e terceiro (Rio Grande do Sul) Estados brasileiros maiores produtores de soja, a correlação entre as produções estimadas e oficiais apresentou tendência de subestimativa próxima a $2 \%$ e boa precisão (Figura 1). O maior desvio ocorreu na safra 2004/2005, para o Estado do Rio Grande do Sul, ano de ocorrência de severa estiagem, ao longo do mês de janeiro, que coincidiu com o estádio da floração (R2) ao de enchimento de grãos (R5), fases de maior sensibilidade à deficiência hídrica. A escassez das chuvas reduziu drasticamente a produtividade das lavouras de soja no Estado, com média de $641 \mathrm{~kg} \mathrm{ha}^{-1}$ (Conab, 2006b). A produtividade média estimada pelo SPSoja-Agritempo, para a referida safra, foi de $1.169 \mathrm{~kg} \mathrm{ha}^{-1}$. Apesar de ser uma média expressivamente inferior à produtividade normalmente registrada no Rio Grande do Sul, supera amplamente o valor apontado pelo levantamento subjetivo da Conab. Apesar do erro, a queda na produtividade, em consequiência da deficiência hídrica, apontou uma importante característica qualitativa do SPSojaAgritempo. Em anos secos, à medida que a perda de produtividade se intensifica, nota-se um descolamento cada vez mais pronunciado entre a estimativa do sistema e o valor observado no campo. Infere-se que essa tendência está associada ao abandono das áreas de produção pelo agricultor à medida que as perdas aumentam, numa tentativa de minimizar seu prejuízo

Tabela 1. Análise estatística entre as produções estaduais estimadas (SPSoja-Agritempo) e oficiais (Conab), pelo teste $t$, com as respectivas probabilidades $\mathrm{P}(\mathrm{T} \leq \mathrm{t})$ e correlações de Pearson (r).

\begin{tabular}{lcc}
\hline Estados & $\mathrm{P}(\mathrm{T} \leq \mathrm{t})^{(1)}$ & $\mathrm{r}$ \\
\hline Rio Grande do Sul & 0,787 & 0,925 \\
Santa Catarina & 0,894 & 0,471 \\
Paraná & 0,891 & 0,805 \\
São Paulo & 0,975 & 0,802 \\
Minas Gerais & 0,976 & 0,946 \\
Mato Grosso do Sul & 0,474 & 0,878 \\
Mato Grosso & 0,908 & 0,951 \\
Goiás & 0,884 & 0,922 \\
Tocantins & 0,572 & 0,993 \\
Bahia & 0,923 & 0,285 \\
Maranhão & 0,973 & 0,949 \\
Piauí & 0,927 & 0,894 \\
\hline
\end{tabular}

(1)Valores maiores que 0,05 indicam que não há diferença significativa entre os valores estimados e de referência, pelo teste t, a 5\% de probabilidade. financeiro com as lavouras. Com isso, outros fatores, além da deficiência hídrica, passam a deplecionar a produtividade das lavouras de soja, o que explica a divergência entre os dados estimados e os dados do levantamento da Conab.

Nas safras 2000/2001 e 2002/2003, no Estado do Rio Grande do Sul, e nas de 2002/2003 em Santa Catarina, observou-se tendência inversa. Nesses anos agrícolas, o volume elevado e a boa distribuição das chuvas foram suficientes para que a umidade do solo atendesse satisfatoriamente à exigência hídrica das lavouras de soja ao longo de todo o ciclo de desenvolvimento, tendose registrado produtividade de $2.395 \mathrm{~kg} \mathrm{ha}^{-1} \mathrm{em} 2000 /$

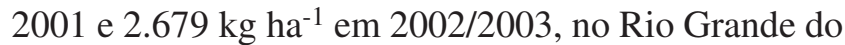
Sul, e de $2.887 \mathrm{~kg} \mathrm{ha}^{-1}$, na safra 2002/2003, no Estado de Santa Catarina (Conab, 2006b). Em condição hídrica satisfatória à cultura da soja, o SPSoja-Agritempo mostra-se demasiadamente sensível à mínima deficiência hídrica e gera médias de produtividade $16 \%$ inferiores no Rio Grande do Sul, de $2.055 \mathrm{~kg} \mathrm{ha}^{-1} \mathrm{e}$ de $2.225 \mathrm{~kg} \mathrm{ha}^{-1}$, nas safras 2000/2001 e 2002/2003, respectivamente. Para o Estado de Santa Catarina, a subestimativa da produtividade foi de $30 \%$ para a safra de 2002/2003, retornando $2.044 \mathrm{~kg} \mathrm{ha}^{-1}$.

No Estado do Paraná, a oscilação da produtividade de soja estimada pelo SPSoja-Agritempo, para todas as safras avaliadas, acompanha o desempenho das lavouras do Estado, tomando-se como referência a produtividade média levantada pela Conab. As maiores discrepâncias foram observadas na safra 2001/2002, em que a produtividade estimada, de $3.192 \mathrm{~kg} \mathrm{ha}^{-1}$, supera em $10,6 \%$ a média oficial de $2.886 \mathrm{~kg} \mathrm{ha}^{-1}$, além da safra 2003/2004, em que a produtividade estimada subestima o dado referencial da Conab em 6\%.

A correlação entre produção estimada e oficial, das safras 2000/2001 a 2005/2006 para a Região Sudeste, apresentou subestimativa inferior a $0,5 \%$ (Figura 2). As maiores discrepâncias, entre produtividade estimada e valor oficial proveniente de levantamento da Conab, ocorreram para as safras 2000/2001 e 2001/2002 para São Paulo, e 2001/2002 para Minas Gerais. Os valores de produtividade, estimadas pelo SPSoja-Agritempo, de $2.865 \mathrm{~kg} \mathrm{ha}^{-1}(2000 / 2001)$ e $2.898 \mathrm{~kg} \mathrm{ha}^{-1}(2001 / 2002)$, para o Estado de São Paulo superestimam em 9,25\% e $7,17 \%$ os valores divulgados pela Conab, para as respectivas safras. Moraes et al. (1998), ao avaliar o desempenho de modelos agrometeorológicos, baseados na penalização da produtividade por deficiência hídrica 
para algumas safras de soja na região de Ribeirão Preto, SP, constataram que o modelo proposto por Doorenbos \& Kassam (1979), o mesmo incorporado ao SPSojaAgritempo, também tendeu a superestimar a produtividade medida em campo. Da mesma forma, para o Estado de Minas Gerais, a produtividade média de $3.054 \mathrm{~kg} \mathrm{ha}^{-1}$ superestimou em $14 \%$ o valor oficial $\left(2.680 \mathrm{~kg} \mathrm{ha}^{-1}\right)$. Não foram observados problemas relacionados à penalização da produtividade, em períodos de deficiência hídrica mais acentuada, menos freqüentes nesses estados. Para as demais safras, as diferenças entre produtividade estimada e oficial foram inferiores a $8 \%$. Os melhores ajustes foram obtidos para os dois estados, na safra 2002/2003, quando as chuvas ocorreram com volume elevado e boa distribuição.
Região Sul (RS, SC, e PR )

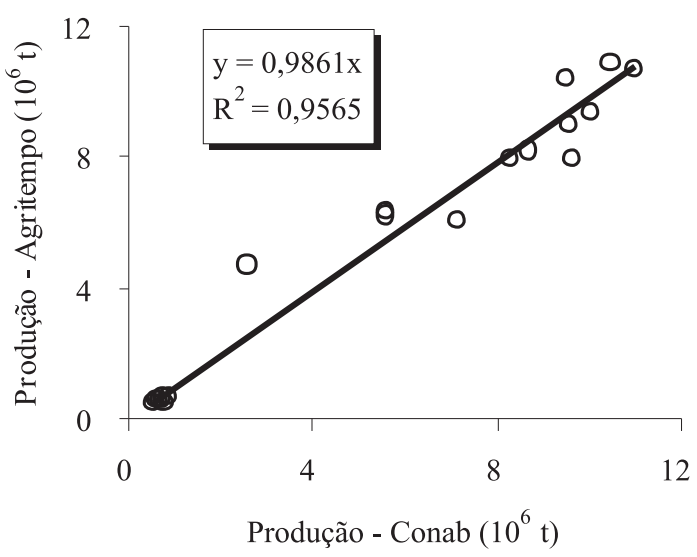

Santa Catarina

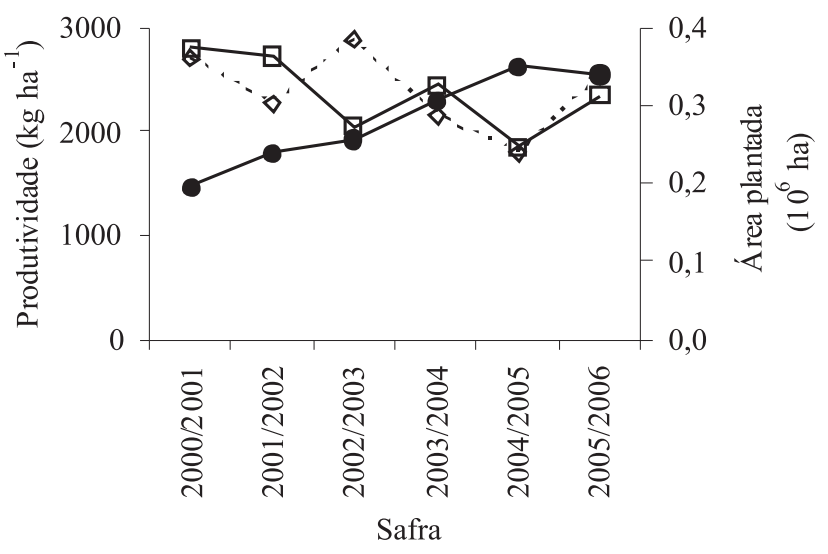

$\square$ Agritempo $\cdots \diamond \cdot$ Conab $\longrightarrow$ Área plantada
Rio Grande do Sul

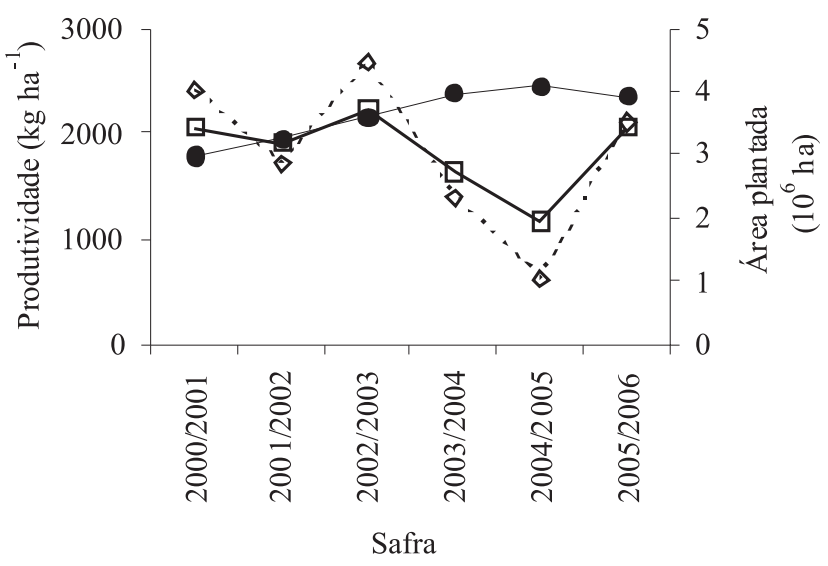

$\square$ Agritempo $\cdots \cdot$ Conab $\longrightarrow$ Área plantada

Paraná

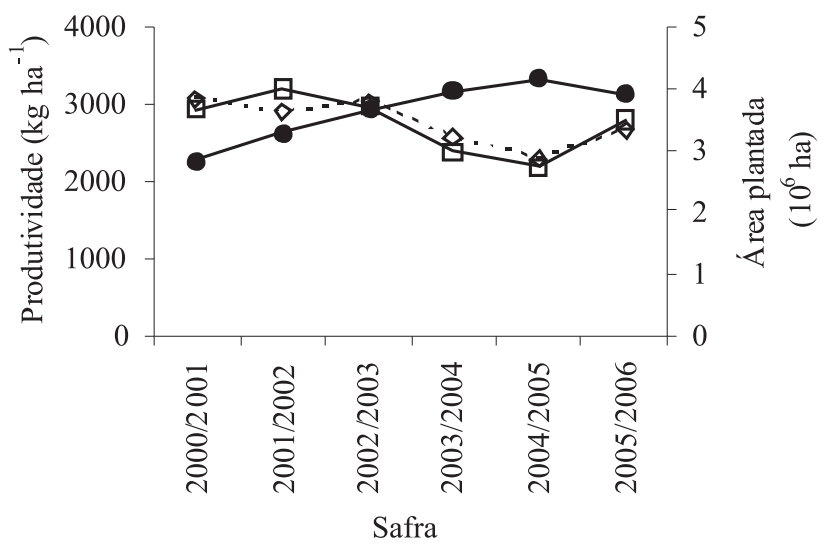

$\square$ Agritempo $\diamond$ Conab $\longrightarrow$ Área plantada

Figura 1. Correlação entre produção de soja estimada (SPSoja-Agritempo) e oficial (Conab), para a Região Sul, variação da produtividade estimada (SPSoja-Agritempo) e de referência (Conab), e área plantada de soja, para os estados do Rio Grande do Sul, Santa Catarina e Paraná, da safra 2000/2001 a 2005/2006. 

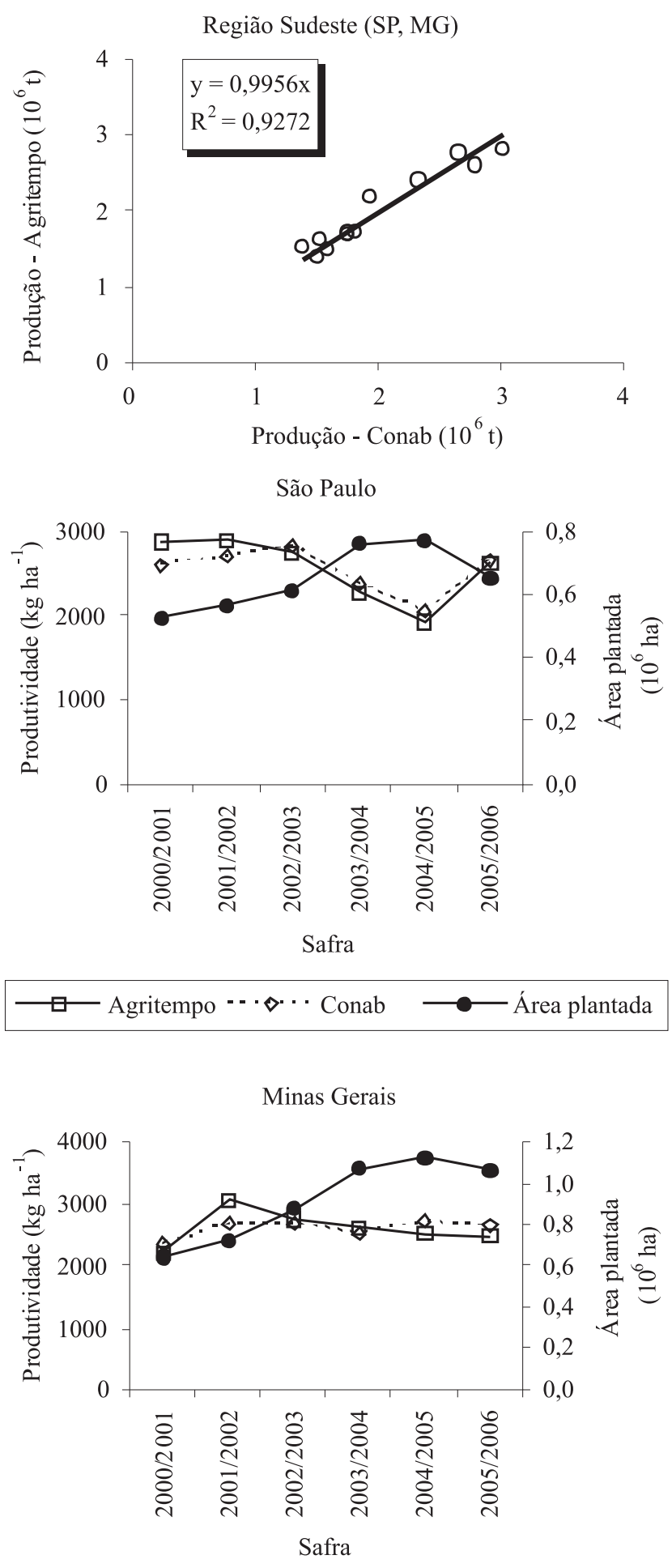

$\longrightarrow$ Agritempo $\cdots \diamond-$ Conab — Área plantada

Figura 2. Correlação entre produção de soja estimada (SPSojaAgritempo) e oficial (Conab) para a Região Sudeste, variação da produtividade estimada (SPSoja-Agritempo) e de referência (Conab), e área plantada de soja, para os estados de São Paulo e Minas Gerais, da safra 2000/2001 a 2005/2006.
Assim como verificado para a Região Sudeste, bom ajuste foi obtido entre produção estimada e oficial para as Regiões Centro-Oeste e Norte (Figura 3), resultado extremamente importante, pois a região Centro-Oeste representa aproximadamente $50 \%$ da produção de soja do Brasil, sendo o Mato Grosso o maior produtor nacional. Nesse Estado, foram obtidos os menores desvios entre produtividade estimada e de referência, para as seis safras avaliadas, com diferença máxima de $7,9 \%$ na safra 2002/2003. Coincidentemente, houve redução drástica da produtividade das lavouras de soja do norte de Mato Grosso, nessa mesma safra, relativa à detecção tardia da incidência da ferrugem asiática da soja (Phakopsora pachyrhizi) (Andrade \& Andrade, 2002), fator deplecionador da produtividade, que não é considerado pelo SPSoja-Agritempo. Na safra seguinte, 2003/2004, houve subestimativa de $12,5 \%$, ano em que o SPSoja-Agritempo, assim como o ocorrido na Região Sul, apresentou sensibilidade excessiva à deficiência hídrica, o que penaliza em demasia a produtividade.

As estimativas para o Mato Grosso do Sul resultaram nos desvios mais acentuados da Região Centro-Oeste, tendo-se observado duas tendências ao longo das safras 2000/2001 a 2005/2006. A produtividade estimada entre as safras 2000/2001 e 2003/2004 esteve sempre abaixo da referência, com subestimativa média de $25 \%$. Para as safras 2004/2005 e 2005/2006, os resultados obtidos foram opostos e a produtividade estimada passou a superar em 19,5\% a produtividade oficial. Como uma das causas para essas discrepâncias tem-se a pequena rede de estações meteorológicas no Estado, fator considerado essencial para o bom desempenho do sistema.

Similar aos resultados obtidos para Mato Grosso, a produtividade estimada manteve-se próxima ao valor de referência no Estado de Goiás, 40 maior produtor de soja do país. O maior desvio ocorreu na safra 2003/2004, quando a produtividade estimada foi de $2.653 \mathrm{~kg} \mathrm{ha}^{-1}$, $11 \%$ superior ao valor de referência $\left(2.390 \mathrm{~kg} \mathrm{ha}^{-1}\right)$.

As diferenças entre produtividade estimada e de referência, para o Tocantins, foram reduzidas progressivamente entre as safras 2000/2001, quando houve superestimativa de cerca de $20 \%$, e 2002/2003, com $1 \%$ de subestimativa. $\mathrm{O}$ desvio médio para as safras seguintes foi inferior a $6,3 \%$.

As maiores discrepâncias verificadas entre produtividade estimada e oficial ocorreram para os estados produtores da Região Nordeste: Bahia, Maranhão e Piauí. A resposta do SPSoja-Agritempo para 
Região Centro-Oeste e Norte ( MS, MT, GO e TO)

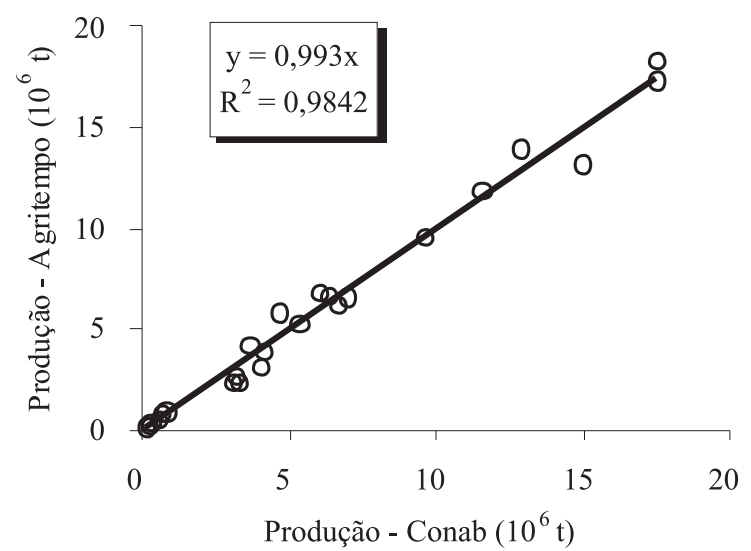

Mato Grosso do Sul

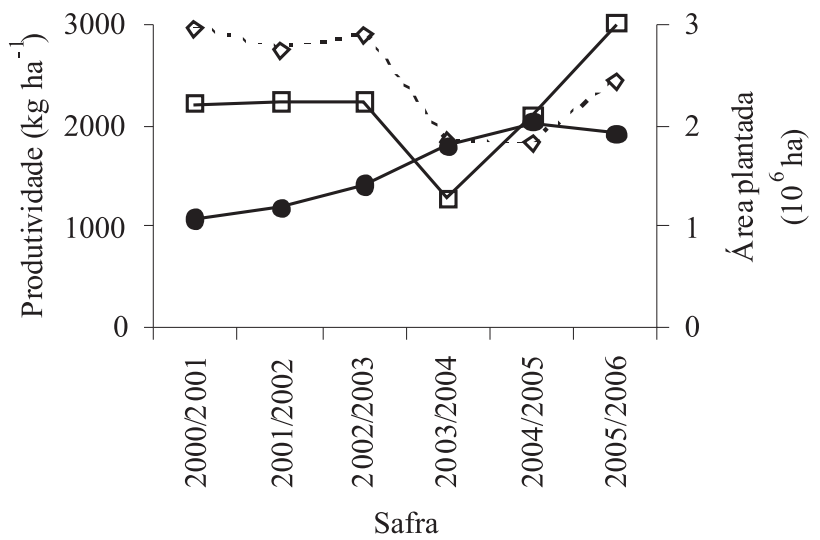

$\square$ Agritempo $\cdots \diamond \cdots$ Conab — Área plantada

Goiás

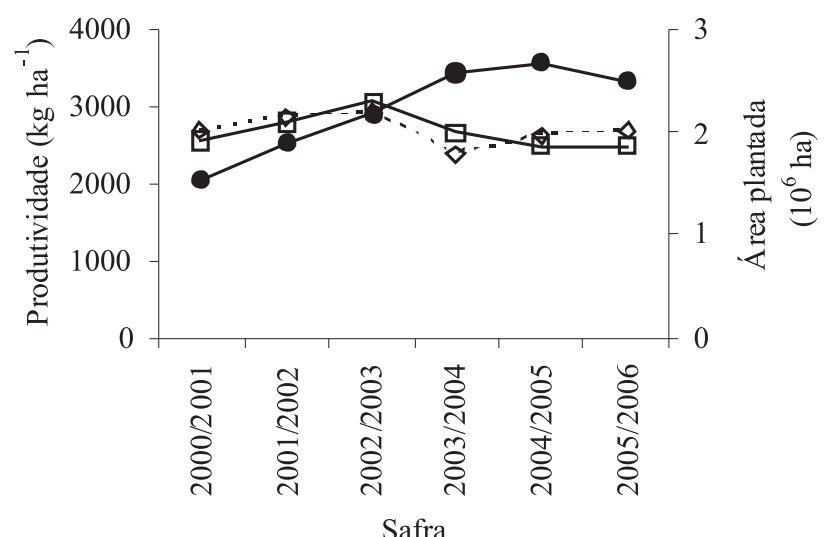

$\square$ Agritempo $\diamond \quad$ Conab $\longrightarrow$ Área plantada
Mato Grosso

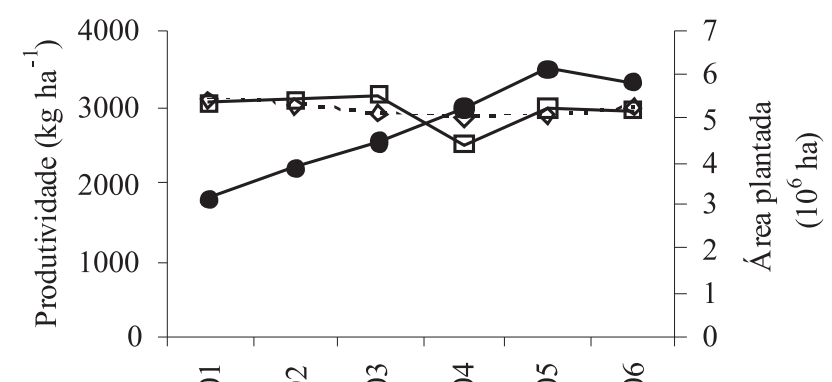

Safra

$\longrightarrow$ Agritempo $\cdots \diamond \cdot-$ Conab —-Área plantada

Tocantins

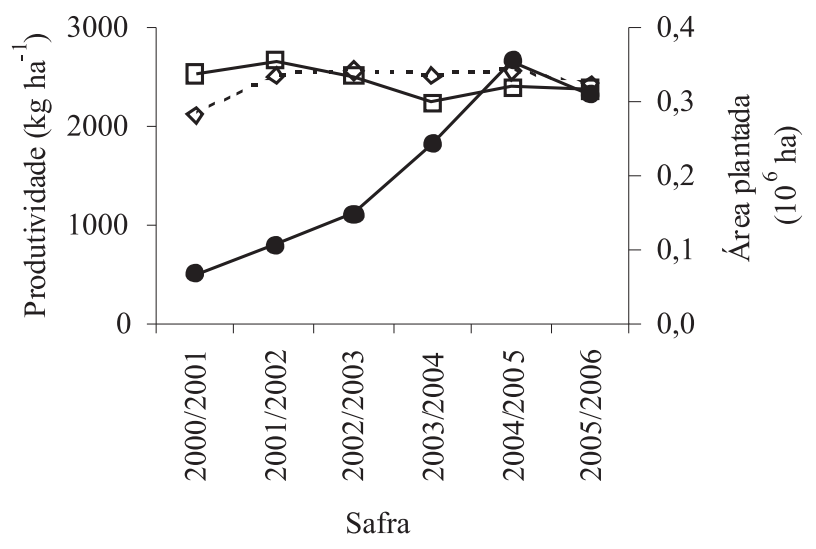

$\square$ Agritempo $\cdots-\cdots$ Conab $\longrightarrow$ Área plantada

Figura 3. Correlação entre produção de soja estimada (SPSoja-Agritempo) e oficial (Conab) para a Regiões Centro-Oeste e Norte, variação da produtividade estimada (SPSoja-Agritempo) e de referência (Conab), e área plantada de soja dos estados de Goiás, Mato Grosso do Sul, Mato Grosso e Tocantins, da safra 2000/2001 a 2005/2006. 
esses três estados foi razoável e sem tendência clara. Para o Estado da Bahia, os desvios oscilaram entre superestimativa de $31 \%$, na safra $2002 / 2003$, e subestimativa de 32\% em 2005/2006 (Figura 4). Os resultados obtidos para o Maranhão, mais satisfatórios que os verificados para o Estado da Bahia, apresentaram superestimativa de 19\%, na safra 2001/2002, e subestimativa de $11 \%$ na safra 2005/2006.

As estimativas do SPSoja-Agritempo no Piauí, entre as safras 2000/2001 e 2003/2004, diferiram expressivamente dos valores de referência na safra 2001/2002, quando houve superestimativa de $133 \%$. Os desvios para as safras 2000/2001 e 2003/2004 foram opostos, tendo superestimado em $36 \%$ a produtividade na primeira safra, e subestimado em $33 \%$ a produtividade oficial na segunda safra. A divergência nas tendências e os altos desvios observados apontam a necessidade de novos ajustes no módulo de penalização e espacialização, além da expansão da rede de estações meteorológicas de superfície.
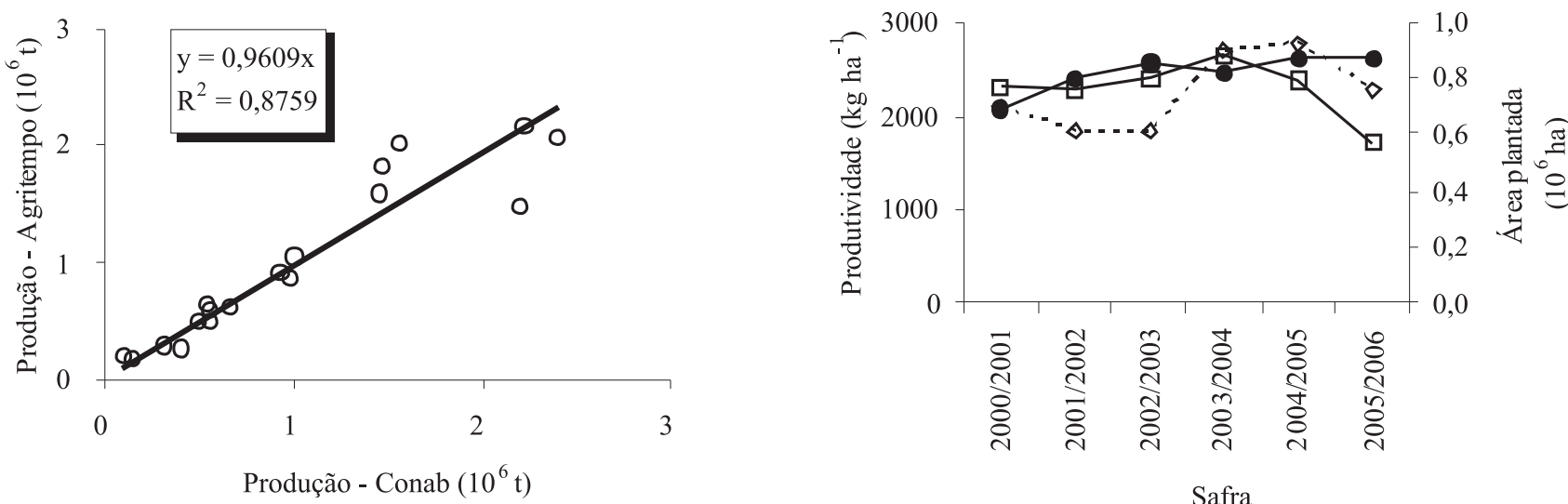

$\square$ Agritempo $\cdots \bullet-$ - Conab —- Área plantada

Maranhão

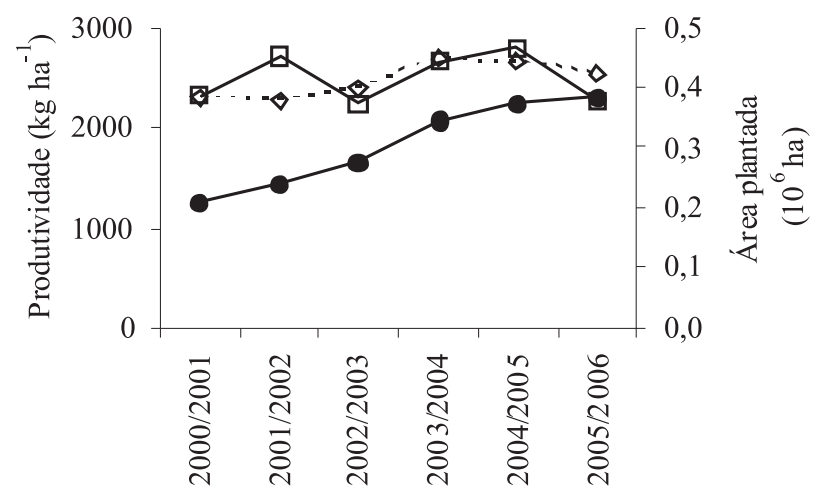

Safra

$\square$ Agritempo $\cdots \diamond \cdots \cdot$ Conab

Área plantada

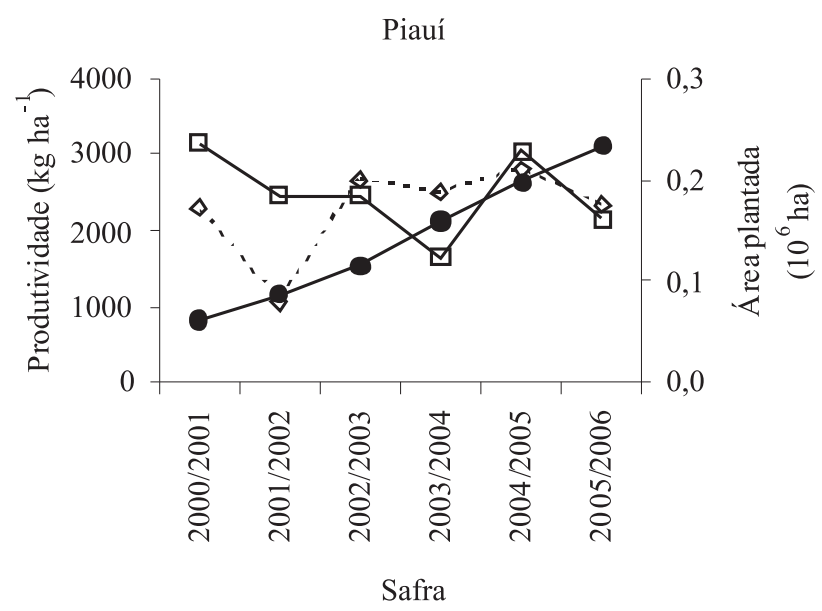

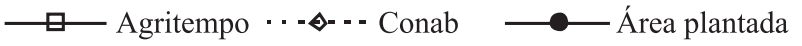

Figura 4. Correlação entre produção de soja estimada (SPSoja-Agritempo) e oficial (Conab) para a Região Nordeste, variação da produtividade estimada (SPSoja-Agritempo) e de referência (Conab), e área plantada de soja dos estados da Bahia, Maranhão e Piauí, da safra 2000/2001 a 2005/2006. 
Durante a safra de soja do ano agrícola de 2005/ 2006, realizaram-se três estimativas com o SPSojaAgritempo, concomitantemente às divulgações do $3 \underline{0}$ (janeiro de 2006), 4o (março de 2006) e 6o (maio de 2006) levantamentos de avaliação da safra 2005/2006 realizados pela Conab, com destaque para a tendência de subestimativa do SPSoja-Agritempo, nos estados do Nordeste (Figura 5). Considerando-se o 60 levantamento, feito em maio de 2006, as subestimativas foram de $16 \%$ para a Bahia, $25 \%$ para o Maranhão e 28\% para o Piauí (Figura 5 C).

Destaca-se, ainda, ao longo das três avaliações, a produção estimada para o Estado do Mato Grosso do Sul. Da primeira estimativa, em janeiro de 2006, à terceira, feita em maio, a produção estimada esteve sempre acima do valor de referência (Figura 5). Essa tendência é atribuída ao efeito associado da severa estiagem ocorrida no sul do estado - fator

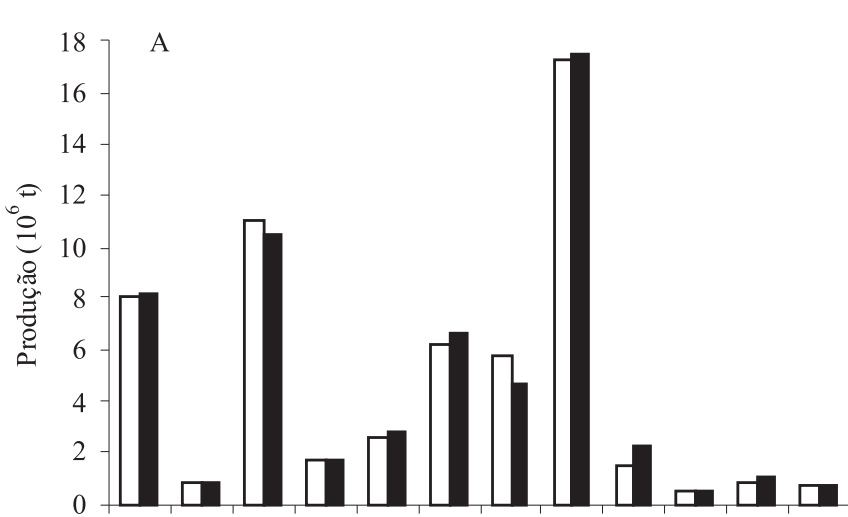

RS SC PR SP MG GO MS MT BA PI MA TO

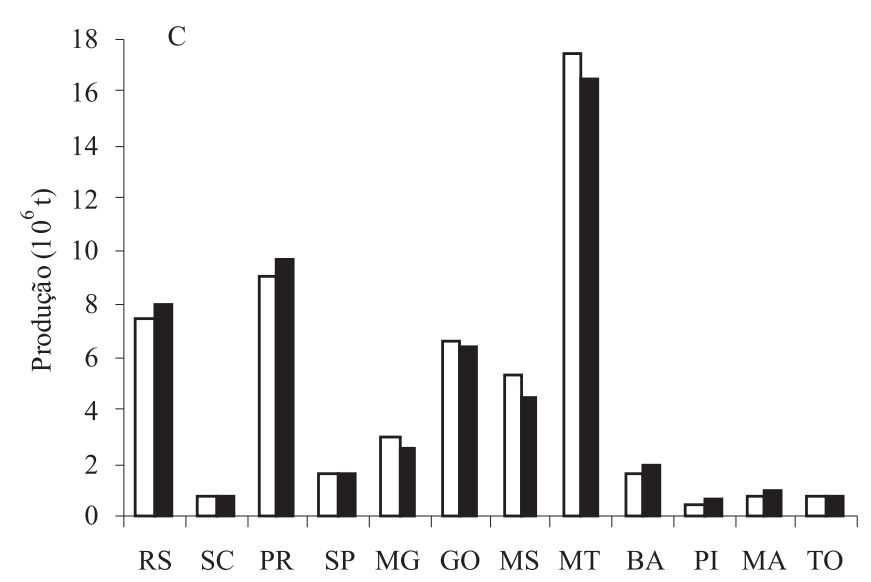

$\square$ Agritempo $\square$ Conab

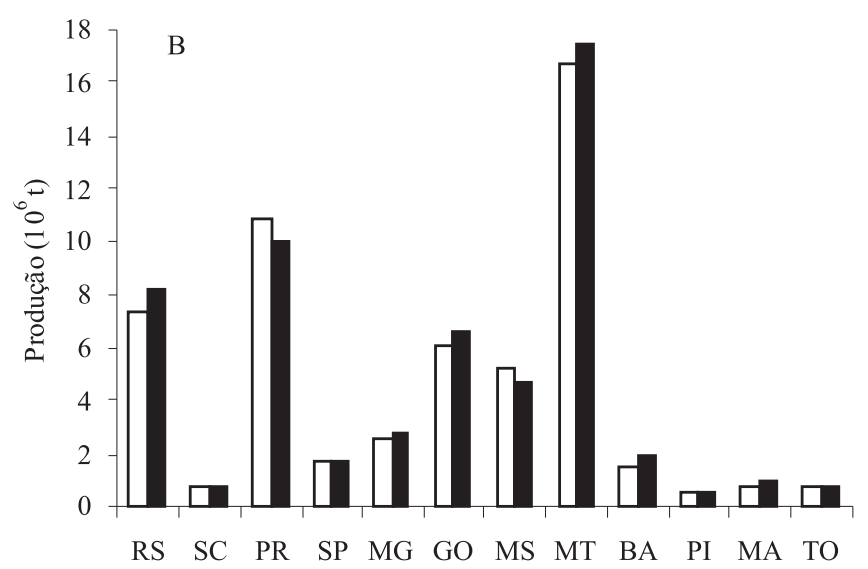

$\square$ Agritempo $\square$ Conab

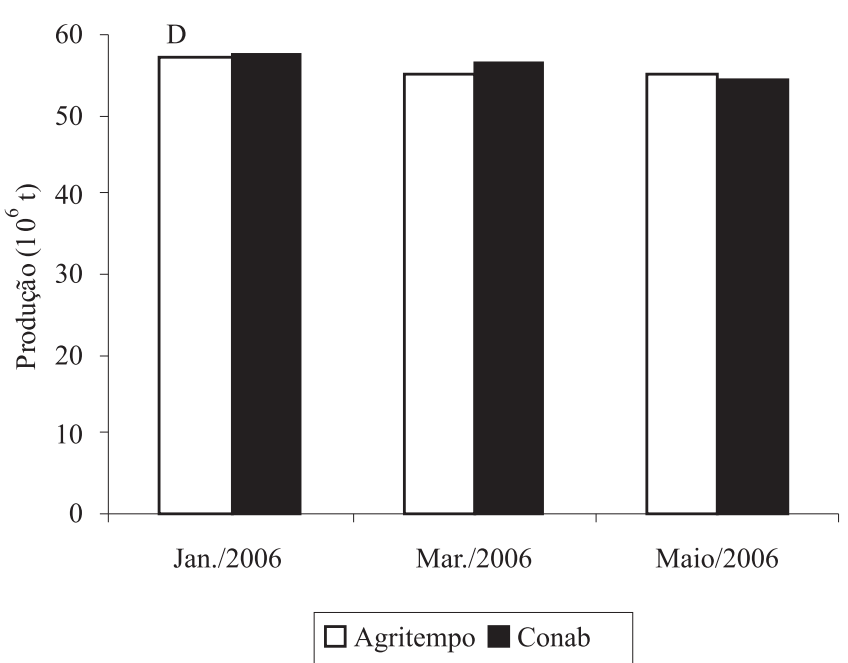

Figura 5. Estimativas das produções estaduais de soja, para a safra 2005/2006, realizadas nos meses de janeiro, março e maio de 2006, em comparação aos valores oficiais de produção por Estado, divulgados nos 3o (A), 4o (B) e 6o levantamentos (C) de avaliação da safra 2005/2006 realizados pela Conab. Comparação entre as produções totais, estimadas nos meses de janeiro, março e maio de 2006, e os valores de referência divulgadas nos 3o , 4ํ e 6o levantamentos de avaliação da safra 2005/2006 realizados pela Conab $(\mathrm{D})$. 
considerado no modelo utilizado no sistema - com a ocorrência de ferrugem asiática - fator não contemplado nas estimativas. Apesar de a produção estimada pelo SPSoja-Agritempo ter apresentado redução de 500.000 t, entre a $1 \underline{a}$ e $3 \underline{a}$ estimativas, resultados das condições adversas do tempo, ainda assim permaneceu a tendência de superestimativa de cerca de $20 \%$, em relação aos valores de referência, e não se atingiram as reais perdas em produção.

A previsão de produção nacional de soja apresentou tendência de queda entre o $1^{\underline{a}}$ e a $3^{\underline{a}}$ simulação, com boa coerência em relação aos levantamentos da Conab. $\mathrm{Na} 2^{\mathrm{a}}$ estimativa, a previsão de produção nacional de soja, obtida com o SPSoja-Agritempo, apresentavase mais coerente com aquela divulgada no 6o levantamento de avaliação da safra 2005/2006, em maio de 2006 (Figura 5). Ao se agruparem as estimativas estaduais, nota-se que o ajuste é expressivamente melhorado, com desvio máximo de $-5,81 \%$ na safra 2000/2001. Evidencia-se a proximidade entre os valores de produção de soja estimada e a divulgada pela Conab, para a safra 2005/2006, valor referente ao 6o levantamento de avaliação da safra 2005/2006, com diferença de apenas $0,62 \%$ (Figura 6).

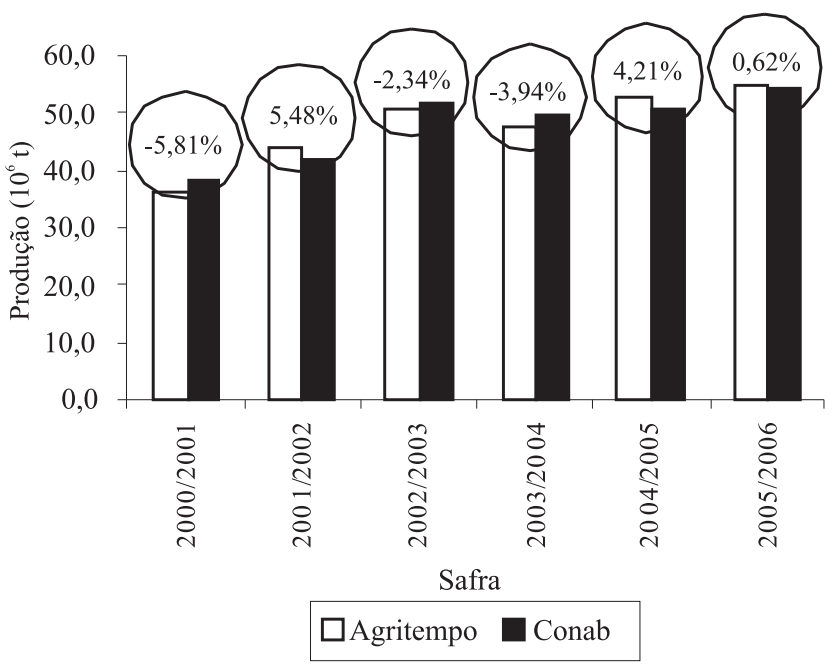

Figura 6. Comparações entre as produções estimadas pelo SPSoja-Agritempo, para o Brasil, e os valores de referência divulgados pela Conab, referentes às safras 2000/2001 a 2005/ 2006, com os respectivos desvios de estimativa.

\section{Conclusão}

O SPSoja-Agritempo apresenta bom desempenho na previsão da safra de soja, nos estados do Mato Grosso, Paraná, São Paulo, Minas Gerais, Tocantins e Goiás, e desempenho inferior na previsão da safra de soja, nos estados do Rio Grande do Sul, Santa Catarina, Mato Grosso do Sul, Maranhão, Piauí e Bahia.

\section{Referências}

ACOCK, B.; ACOCK, M.C. Potential for using long-term fieldresearch data to develop and validate crop simulators. Agronomy Journal, v.83, p.56-61, 1991.

ACOCK, B.; TRENT, A. The soybean crop simulator, GLYCIM: documentation for the modular version 91. Moscow: University of Idaho, Department of Plant, Soil and Entomological Sciences, 1991. 242p.

ALAGARSWAMY, G.; SINGH, P.; HOOGENBOOM, G.; WANI, S.P.; PATHAK, P.; VIRMANI, S.M. Evaluation and application of the CROPGRO-soybean simulation model in a Vertic Inceptisol. Agricultural Systems, v.63, p.19-32, 2000.

ANDRADE, P.J.M.; ANDRADE, D.F. de A.A. Ferrugem asiática: uma ameaça à sojicultura brasileira. Dourados: Embrapa Agropecuária Oeste; Chapadão do Sul: Fundação Chapadão, 2002. 11p. (Embrapa Agropecuária Oeste. Circular técnica, 11).

ANTUNES, J.F.G. Aplicação de lógica fuzzy para estimativa de área plantada da cultura de soja utilizando imagens AVHRRNOAA. 2005. 91p. Dissertação (Mestrado) - Universidade Estadual de Campinas, Campinas.

BERKA, L.M.S.; RUDORFF, B.F.T.; SHIMABUKURO, Y.E. Soybean yield estimation by an agrometeorological model in a GIS. Scientia Agricola, v.60, p.433-440, 2003.

BOARD, J.E. A regression model to predict soybean cultivar yield performance at late planting dates. Agronomy Journal, v.94, p.483492, 2002.

BOARD, J.E.; ZHANG, W.; HARVILLE, B.G. Yield rankings for soybean cultivars grown in narrow and wide rows with late planting dates. Agronomy Journal, v.88, p.240-245, 1996.

CHEEROO-NAYAMUTH, B.F. Crop modelling/simulation: an overview. In: ANNUAL MEETING OF AGRICULTURAL SCIENTISTS, 1999, Réduit. [Proceedings]. Réduit: Food and Agricultural Research Council, 1999. p.11-26.

CONAB. Sexto levantamento de avaliação da safra 2005/2006. Brasília, 2006b. 22p. Disponível em: <http://www.conab.gov.br/ conabweb/download/safra/6_levgraos_maio06.pdf $>$. Acesso em: 28 maio 2006.

CONAB. Soja - Brasil: série histórica de área plantada: safras 1976/ 77 a 2006/07. Brasília, 2006a. Disponível em: <http:// www.conab.gov.br/conabweb/download/safra/SojaSerieHist.xls $>$. Acesso em: 10 jan. 2006.

DOORENBOS, J.; KASSAM, A.H. Yield response to water. Rome: FAO, 1979. 193p. (Irrigation and drainage paper, 33). 
EPIPHANIO, J.C.N.; LUIZ, A.J.B.; FORMAGGIO, A.R. Estimativa de áreas agrícolas municipais, utilizando sistema de amostragem simples sobre imagens de satélite. Bragantia, v.61, p.187-197, 2002.

FERRAUDO, A.S.; ANDRÉ, R.G.B.; PINHO, S.Z. de. Modelo agrometeorológico para estimar rendimento de grãos de milho. Revista Brasileira de Agrometeorologia, v.3, p.93-96, 1995.

FIGUEIREDO, D.C. Projeto GeoSafras - aperfeiçoamento do sistema de previsão de safras da CONAB. Revista de Política Agrícola, v.14, p.110-120, 2005.

FONTANA, D.C.; BERLATO, M.A.; LAUSCHNER, M.H.; MELLO, R.W. de. Modelo de estimativa de rendimento de soja no Estado do Rio Grande do Sul. Pesquisa Agropecuária Brasileira, v.36, p.399-403, 2001.

GARCIA-PAREDES, J.D.; OLSON, K.R.; LANG, J.M. Predicting corn and soybean productivity for Illinois soils. Agricultural Systems, v.64, p.151-170, 2000.

HOOGENBOOM, G.; JONES, J.W.; WILKENS, P.W.; BATCHELOR, W.D.; BOWEN, W.T.; HUNT, L.A.; PICKERING, N.B.; SINGH, U.; GODWING, D.C.; BAER, B.; BOOTE, K.J.; RITCHIE, J.T.; WHITE, J.W. Crops models. In: TSUJI, G.Y.; UEHARA, G.; BALAS, S. (Ed.). DSSAT: decision support system for agrotechnology transfer. Version 3. Honolulu: University of Hawaii, 1994. v.2. p.95-244.

JENSEN, M.E. Water consumption by agricultural plants. In: KOZLOWSKY, T.T. (Ed.). Water deficits and plant growth. New York: Academic Press, 1968. v.2. p.1-22.

LUIZ, A.J.B.; OLIVEIRA, J.C.; EPIPHANIO, J.C.N.; FORMAGGIO, A.R. Auxílio das imagens de satélite aos levantamentos por amostragem em agricultura. Agricultura em São Paulo, v.49, p.49-54, 2002.

MORAES, A.V. de C.; CAMARGO, M.B.P. de; MASCARENHAS, H.A.A.; MIRANDA, M.A.C. de; PEREIRA, J.C.V.N.A. Teste e análise de modelos agrometeorológicos de estimativa de produtividade para a cultura da soja na região de Ribeirão Preto. Bragantia, v.57, p.393-406, 1998.

PASSIOURA, J.B. Simulation models: science, snake oil, education, or engineering? Agronomy Journal, v.88, p.690-694, 1996.

PEDERSEN, P.; LAUER, J.G. Application of the CROPGROsoybean model to predict soybean yield. In: FERTILIZER, AGLIME
AND PEST MANAGEMENT CONFERENCE, Madison. Proceedings. Madison: University of Wisconsin, 2002. p.186-189.

POPP, M.P.; DILLON, C.R.; KEISLING, T.C. Economic and weather influences on soybean planting strategies on heavy soils. Agricultural Systems, v.76, p.969-984, 2003.

REDDY, V.R.; ACOCK, B.; WHISLER, F.D. Crop management and input optimization with GLYCIM: differing cultivars. Computers and Electronics in Agriculture, v.13, p.37-50, 1995.

REUNIÃO DE PESQUISA DE SOJA DA REGIÃO CENTRAL DO BRASIL, 26., 2004, Ribeirão Preto. Ata. Londrina: Embrapa Soja: Fundação Meridional, 2004. 272p. (Embrapa Soja. Documentos, 238).

RIZZI, R.; RUDORFF, B.F.T. Estimativa da área de soja no Rio Grande do Sul por meio de imagens Landsat. Revista Brasileira de Cartografia, v.57, p.226-234, 2005.

ROMANI, L.A.S.; EVANGELISTA, S.R.M.; ZULLO JUNIOR, J.; AGUIAR, D.A.; FONSECA, M.F. Geração de mapas agrometeorológicos em tempo real via Internet. In: CONGRESSO BRASILEIRO DA SOCIEDADE BRASILEIRA DE INFORMÁTICA APLICADA À AGROPECUÁRIA E AGROINDÚSTRIA, 4., 2003, Porto Seguro. Anais. Lavras: SBIAgro, 2003. p.1-4. 1 CD-ROM.

RUDORFF, B.F.T.; BERKA, L.M.S.; MOREIRA, M.A.; DUARTE, V.; XAVIER, A.C.; ROSA, V.G.C.; SHIMABUKURO, Y.E. Imagens de satélite no mapeamento e estimativa de área de canade-açúcar em São Paulo: ano-safra 2003/04. Agricultura em São Paulo, v.52, p.21-39, 2005.

RUÍZ-NOGUEIRA, B.; BOOTE, K.J.; SAU, F. Calibration and use of CROPGRO-soybean model for improving soybean management under rainfed conditions. Agricultural Systems, v.68, p.151-173, 2001.

SAU, F.; BOOTE, K.J.; RUÍZ-NOGUEIRA, B. Evaluation and improvement of CROPGRO-soybean model for a cool environment in Galicia, Northwest Spain. Field Crops Research, v.61, p.273$291,1999$.

SINCLAIR, T.R. Water and nitrogen limitations in soybean grain production. I. Model development. Field Crops Research, v.15, p.125-141, 1986.

THORNTHWAITE, C.W. An approach toward a rational classification of climate. Geographical Review, v.38, p.55-94, 1948.

THORNTHWAITE, C.W.; MATHER, J.R. The water balance. Centerton: Laboratory of Climatology, 1955. 104p.

Recebido em 22 de junho de 2006 e aprovado em 8 de março de 2007 